\title{
Risk of septic arthritis in patients with rheumatoid arthritis and the effect of anti-TNF therapy: results from the British Society for Rheumatology Biologics Register
}

\author{
J B Galloway, ${ }_{1}$ K L Hyrich, ${ }^{1}$ L K Mercer, ${ }^{1}$ W G Dixon, ${ }^{1}$ A P Ustianowski, ${ }^{2}$ M Helbert, ${ }^{3}$ \\ K D Watson, ${ }^{1}$ M Lunt, ${ }^{1}$ D P M Symmons ${ }^{1}$; on behalf of the BSR Biologics Register
}

- Additional data are published online only. To view these files please visit the journal online at (http://ard.bmj.com).

${ }^{1}$ Arthritis Research UK Epidemiology Unit, Manchester Academic Health Sciences Centre, University of Manchester, Manchester, UK 2Department of Infectious Diseases, North Manchester General Hospital, Manchester, UK ${ }^{3}$ Department of Immunology, Central Manchester NHS Foundation Trust, Manchester, UK

\section{Correspondence to} Professor D P M Symmons, Arthritis Research UK Epidemiology Unit, Manchester Academic Health Sciences Centre, University of Manchester, Stopford Building, Oxford Road, Manchester M13 9PT, UK deborah.symmons@ manchester.ac.uk

Accepted 12 June 2011 Published Online First 21 July 2011

\section{ABSTRACT}

Objectives To evaluate the risk of septic arthritis (SA) in patients with rheumatoid arthritis (RA) treated with antitumour necrosis factor (TNF) therapy.

Methods Using data from the British Society for Rheumatology Biologics Register, a prospective observational study, the authors compared the risk of SA between 11881 anti-TNF-treated and 3673 non-biological disease-modifying antirheumatic drug (nbDMARD)treated patients.

Results 199 patients had at least one episode of SA (anti-TNF: 179, nbDMARD: 20). Incidence rates were: anti-TNF 4.2/1000 patient years (pyrs) follow-up (95\% Cl 3.6 to 4.8 ), nbDMARD 1.8/1000 pyrs (95\% Cl 1.1 to 2.7). The adjusted HR for $S A$ in the anti-TNF cohort was $2.3(95 \% \mathrm{Cl} 1.2$ to 4.4$)$. The risk did not differ significantly between the three agents: adalimumab, etanercept and infliximab. The risk was highest in the early months of therapy. The patterns of reported organisms differed in the anti-TNF cohort. Prior joint replacement surgery was a risk factor for SA in all patients. The rate of postoperative joint infection (within 90 days of surgery) was $0.7 \%$. This risk was not significantly influenced by anti-TNF therapy.

Conclusions Anti-TNF therapy use in RA is associated with a doubling in the risk of SA. Physicians and surgeons assessing the RA patient should be aware of this potentially life-threatening complication.

\section{INTRODUCTION}

Septic arthritis (SA) is a serious medical condition that, even with prompt treatment, can lead to irreversible joint damage and has a death rate of around $10 \% .^{1}$ The incidence of SA in the general population is around 4-10 per 100000 patient years (pyrs) and seems to be rising, ${ }^{2} 3$ probably due to the combination of an ageing population and larger numbers of orthopaedic interventions.

Important risk factors for SA include increasing age, joint prosthesis, skin infection and pre-existing joint damage. ${ }^{4}{ }^{5}$ Patients with rheumatoid arthritis (RA) may have many of these risks combined with the use of immunosuppressive medications. The risk of SA in an RA patient, irrespective of therapy, is increased by $4-15$-fold. ${ }^{5}$ Although one might expect immunosuppressive therapy to increase the risk of SA, this has not been well studied. This question has been of increasing interest over the last decade since the emergence of biological therapies. Anti-tumour necrosis factor (anti-TNF) therapies were the first class of biological agents to become established in routine RA care. Data have emerged suggesting that these drugs confer a small but significant risk of serious infections, especially during the first months of treatment. ${ }^{7-9}$ It is also apparent that this risk differs by anatomical site and that there is increased susceptibility to certain pathogens. ${ }^{9-11}$

There is very limited information regarding the effect of anti-TNF therapy on the risk of SA. Case reports have described patients on anti-TNF therapy developing SA as a multifocal disease or with unusual causative organisms. ${ }^{12-14}$ Although case reports are a useful tool for raising questions, they cannot provide information regarding disease incidence or relative risk. An additional important question relates to the risk of SA following joint replacement surgery in anti-TNF-treated patients.

In 2001, the British Society for Rheumatology (BSR) established a national prospective cohort study of patients starting anti-TNF therapy for RA, the BSR Biologics Register (BSRBR). This is the largest register of its kind worldwide and includes detailed records of serious adverse events including SA occurring in patients receiving anti-TNF therapy as well as in a cohort of RA patients not exposed to anti-TNF therapy.

Our primary aim was to test the hypothesis that anti-TNF therapy increases the risk of SA compared with non-biological disease-modifying antirheumatic drug (nbDMARD) therapy. Secondary analysis considered whether anti-TNF therapy confers additional risk to patients who have joint replacement surgery either prior to starting therapy or during follow-up.

\section{METHODS}

The study commenced in 2001 alongside national recommendations within the UK that all RA patients prescribed anti-TNF therapy should be enrolled with the register. ${ }^{15}$ Patients were recruited to the anti-TNF cohort from 2001 onwards. Three antiTNF agents were licensed for use in the UK during this period, with infliximab (INF) and etanercept (ETN) being available from the start of the study, while the third drug, adalimumab (ADA), came into clinical practice in 2003. Recruitment targets of 4000 patients for the ETN cohort were met in 2005 , for INF in 2007 and for ADA in 2008. Before recruitment targets were met, it was estimated that over $80 \%$ of anti-TNF-treated patients with RA in the UK were registered on the BSRBR. ${ }^{16} \mathrm{~A}$ comparison online under the BMJ Journals unlocked scheme, see http:// ard.bmj.com/info/unlocked.dtl 
cohort of patients with active RA (defined as having a 28-joint count disease activity score (DAS28) >4.2) was recruited in parallel. These patients were receiving an nbDMARD and were biologically naive. Patients prescribed biologics were recruited from across the UK (over 250 hospitals) whereas controls were recruited from 29 centres. These control centres reflect a combination of secondary and tertiary care rheumatology centres distributed across the UK and are listed in full in the BSRBR control centre consortium supplementary data file.

\section{Baseline assessment}

All patients in this study had a physician diagnosis of RA. Baseline information included demographics, disease duration, a measure of self-reported physical function (the Health Assessment Questionnaire (HAQ) ${ }^{17}$ ), DAS28 score, ${ }^{18}$ baseline steroid use, smoking history, baseline comorbidity and surgery, including prior joint replacement. For the purpose of this analysis, we have considered only large joint replacements (shoulder, elbow, hip and knee) because when reviewing reports of small joint surgery, it was difficult to distinguish between soft-tissue procedures and arthroplasty.

\section{Follow-up}

Follow-up information was collected from three sources. First, six monthly questionnaires were sent to the treating rheumatologist for 3 years and annually thereafter. Second, data were collected directly from the patients six monthly for their first 3 years of the study. Patients were provided with a handheld diary card to record details of all hospital attendances as well as new prescriptions. Details of joint replacements during follow-up were collected from these two sources. Third, all patients were flagged with the UK National Health Service Information Centre, which informs the register of any deaths and the causes of deaths. All patients had to have at least one returned consultant follow-up questionnaire prior to 31 December 2009.

\section{Case definition and verification}

This analysis was confined to serious cases of SA. 'Serious' infections were defined as those requiring intravenous antibiotics or hospitalisation, or those resulting in death. All serious infections reported to the BSRBR were followed up with requests for additional information from the treating clinician to gather information on the site of infection and microbiology results. All reported cases of SA were then verified by a BSRBR clinician (JBG).

Events were ascribed to anti-TNF if they occurred while the patient was receiving anti-TNF therapy or within 90 days of the first missed dose. Events were attributed to the most recent drug exposure in patients who switched anti-TNF therapy. Patients were censored from further follow-up after their first episode of SA. Only one case of articular Mycobacterium tuberculosis was reported to the BSRBR, which has been described in a previous publication and is not included in this analysis. ${ }^{19}$

\section{Statistical methods}

Crude incidence rates were calculated as the number of episodes of SA per 1000 pyrs of follow-up. Cumulative hazards were compared between the different cohorts using a NelsonAalen plot. A Cox model was used to calculate HR between the groups. Changes to the incidence rate over time in the antiTNF cohort were analysed using a spline model. Potential confounders were identified prior to the analysis as variables that were either unbalanced between the nbDMARD and anti-TNF cohorts (age, gender, disease duration, DAS28, HAQ, steroid exposure, prior joint replacement, calendar year of entry into the study) or significant predictors of infection (chronic obstructive pulmonary disease, diabetes). Adjustment for these potential confounders was made using propensity scores. The use of this method in observational studies has been described in detail previously. ${ }^{20}$

In a secondary analysis, the influence of joint replacement was examined. Patients were entered into this analysis when they were at risk of a prosthetic infection (ie, from baseline if they had a prior joint replacement or at the date of surgery if they had a joint replacement during follow-up). An adjusted Cox proportional hazards model was used to compare rates between groups. The risk of postoperative infection following joint replacement surgery during active follow-up was analysed separately using adjusted logistic regression to compare the odds of developing an infection within 90 days of joint replacement surgery between anti-TNF and nbDMARD cohorts. Adjustment was made for the same confounders as identified in the primary analysis. Missing baseline data were replaced using multiple imputations. All analysis was done using Stata 10.1 (StataCorp, College Station, Texas, USA).

Further details of the statistical methodology are presented in a data supplement online.

Ethics approval for this study was obtained in December 2000 from the Multicentre Research Ethics Committee for the Northwest of England.

\section{RESULTS}

The baseline characteristics of the 15554 patients included in the analysis are shown in table 1 . Although both anti-TNF and nbDMARD groups had active RA, there were significant differences at baseline. The patients receiving anti-TNF therapy were younger and proportionally more were women. The anti-TNF group had significantly longer disease duration as well as higher disease activity. History of joint replacement was higher in the anti-TNF cohort $(24 \%$ vs $14 \%(p<0.001))$.

Incident SA was reported in 199 patients during the followup period (179 anti-TNF; 20 nbDMARD). In univariate analysis, increasing age, longer disease duration, higher $\mathrm{HAO}$, higher DAS28 score, baseline steroid exposure, prior orthopaedic surgery and diabetes were associated with SA irrespective of antiTNF exposure. The strongest association was with a history of large joint orthopaedic surgery at baseline irrespective of whether the SA developed in a prosthetic joint: HR 2.45 (1.90-3.17).

The incident rate for SA was significantly higher in the antiTNF cohort (4.2/1000 follow-up pyrs) than in the nbDMARD group (1.8/1000 pyrs) (table 2$)$. The cumulative incidence over time is presented in figure 1. The unadjusted HR for SA in the anti-TNF cohort was 2.5 (95\% CI 1.6 to 4.0). After full adjustment, patients on anti-TNF therapy were more than twice as likely to develop SA as the nbDMARD controls: adjusted HR 2.3 (95\% CI 1.2 to 4.4 ).

Hazard estimates for the first year of follow-up were increased in all three anti-TNF agents; however, beyond 1 year, the hazard in the ETN cohort increased more than in the ADA and INF cohorts (figure 1). The adjusted HR was highest within the ETN cohort and lowest for ADA (table 2) although there were no differences between the three individual drugs or between the monoclonal antibody class and ETN that achieved statistical significance (results not shown).

The pattern of joint involvement was similar in the two cohorts with the knee being the most frequent site of infection 
Table 1 Baseline patient characteristics

\begin{tabular}{|c|c|c|c|c|c|c|c|}
\hline Characteristic & $\begin{array}{l}\text { nbDMARD } \\
(\mathrm{n}=3673)\end{array}$ & $\begin{array}{l}\text { All TNF } \\
(n=11881)\end{array}$ & p Value* & $\begin{array}{l}\text { Etanercept } \\
(\mathrm{n}=4139)\end{array}$ & $\begin{array}{l}\text { Infliximab } \\
(\mathrm{n}=3475)\end{array}$ & $\begin{array}{l}\text { Adalimumab } \\
(\mathrm{n}=4267)\end{array}$ & p Value ${ }^{\dagger}$ \\
\hline Age, mean (SD) & $60(12)$ & $56(12)$ & $<0.001$ & $56(12)$ & $56(12)$ & $57(12)$ & 0.018 \\
\hline Sex (\% female) & $2652(72)$ & $9053(76)$ & $<0.001$ & $3193(77)$ & $2626(76)$ & $3234(76)$ & 0.203 \\
\hline DAS28 (mean (SD)) & $5.1(1.3)$ & $6.6(1.0)$ & $<0.001$ & $6.6(1.0)$ & $6.6(1.0)$ & $6.5(1.0)$ & $<0.001$ \\
\hline HAO score (mean (SD)) & $1.5(0.8)$ & $2.0(0.6)$ & $<0.001$ & $2.1(0.6)$ & $2.1(0.5)$ & $1.9(0.6)$ & $<0.001$ \\
\hline Disease duration (years (median (IQR))) & $6(1,15)$ & $11(6-19)$ & $<0.001$ & $12(6-19)$ & $12(6-19)$ & $10(5-18)$ & $<0.001$ \\
\hline Baseline steroid use (n (\%)) & $845(23)$ & $5228(44)$ & $<0.001$ & $1979(48)$ & $1609(46)$ & $1664(39)$ & $<0.001$ \\
\hline Diabetes (n (\%)) & $234(6.7)$ & $675(5.8)$ & 0.033 & $255(6)$ & $169(4)$ & $261(6)$ & 0.026 \\
\hline COPD (n (\%)) & $304(8)$ & $570(5)$ & $<0.001$ & $222(5)$ & $165(5)$ & $183(4)$ & 0.070 \\
\hline \multicolumn{8}{|l|}{ Smoking (n (\%)) } \\
\hline Current & $868(24)$ & $2580(22)$ & 0.001 & $846(21)$ & $757(22)$ & $977(23)$ & 0.029 \\
\hline Ex & $1454(40)$ & $4510(38)$ & & $1576(38)$ & $1314(38)$ & $1620(38)$ & \\
\hline Never & $1333(36)$ & $4714(40)$ & & $1691(41)$ & $1386(40)$ & $1637(39)$ & \\
\hline Prior large joint replacement $(\mathrm{n}(\%))^{\ddagger}$ & $521(14)$ & $2837(24)$ & $<0.001$ & $1090(26)$ & $846(24)$ & $901(21)$ & $<0.001$ \\
\hline $\begin{array}{l}\text { Large joint replacement during follow-up } \\
(\mathrm{n}(\%))^{\ddagger}\end{array}$ & $481(13)$ & $4390(37)$ & $<0.001$ & $1907(46)$ & $1111(32)$ & $1372(32)$ & $<0.001$ \\
\hline
\end{tabular}

${ }^{*}$ Represents the significance of differences between the DMARD and anti-TNF cohorts using $\chi^{2}$ tests for categorical outcomes and Wilcoxon rank sum tests for continuous variables.

${ }^{\dagger}$ Represents the significance of differences between the three anti-TNF drugs using $\chi^{2}$ tests for categorical outcomes and Kruskal-Wallis rank tests for continuous variables.

†Elbow, shoulder, hip, knee and ankle.

Anti-TNF, anti-tumour necrosis factor; COPD, chronic obstructive pulmonary disease; DAS28, 28-joint count disease activity score; HAQ, Health Assessment Questionnaire; nbDMARD, non-biological disease-modifying antirheumatic drug.

Table 2 Risk of septic arthritis in patients with rheumatoid arthritis

\begin{tabular}{|c|c|c|c|c|c|}
\hline & nbDMARD & All anti-TNF & Etanercept & Infliximab & Adalimumab \\
\hline Exposure time (years) & 11426 & 42671 & 18554 & 10827 & 13289 \\
\hline Events $(n)$ & 20 & 179 & 86 & 41 & 52 \\
\hline $\begin{array}{l}\text { Incident rate/1000 pyrs } \\
(95 \% \mathrm{CI})\end{array}$ & $1.8(1.1$ to 2.7$)$ & 4.2 (3.6 to 4.8$)$ & 4.6 (3.7 to 5.7$)$ & $3.8(2.7$ to 5.1$)$ & $3.9(2.9$ to 5.1$)$ \\
\hline Unadjusted HR (95\% Cl) & Ref & 2.5 (1.6 to 4.0$)$ & $3.0(1.8$ to 4.8$)$ & 2.2 (1.3 to 3.8$)$ & $2.3(1.4$ to 3.8$)$ \\
\hline Adjusted HR (95\% CI) & Ref & 2.3 (1.2 to 4.4$)$ & 2.5 (1.3 to 4.9$)$ & 2.4 (1.0 to 5.8$)$ & $1.9(0.9$ to 4.0$)$ \\
\hline
\end{tabular}

Anti-TNF, anti-tumour necrosis factor; nbDMARD, non-biological disease-modifying antirheumatic drug; pyrs, patient years.

(anti-TNF 31\%; nbDMARD 22\%). Microbiological confirmation was available for 94 (53\%) of the anti-TNF cases and 7 (35\%) of the DMARD cases. Staphylococcus aureus was the most frequently reported organism in both cohorts (anti-TNF $57 \%$; DMARD $43 \%$ ). Six (11\%) of the S aureus cases in the anti-TNF cohort were reported as being methicillin resistant. Also, within the anti-TNF cohort, several species that seldom cause joint infections were reported. These included five cases of intracellular infection (two Listeria, three Salmonella) and four cases of Pseudomonas aeruginosa infection. Analysis of results limited to only culture-proven SA yielded an adjusted HR of 2.7 (95\% CI 1.0 to 6.9) for anti-TNF.

The hazard for SA in the anti-TNF cohort was greatest in the early months of therapy (figure 2). This risk then decreased steadily over the remainder of the follow-up period. The 30-day all-cause mortality following SA was 6\% (10/178) in the antiTNF cohort and $10 \%(2 / 20)$ in the DMARD cohort.

Forty-seven (24\%) of the 199 cases were in prosthetic joints. In the patients with a history of large joint replacement (either at baseline or during follow-up), the incidence of prosthetic joint SA was not different between nbDMARD and anti-TNF cohorts (table 3). Thirty of the 47 prosthetic joint SA cases occurred postoperatively (within 90 days of the joint replacement surgery). In total, 4871 large joint replacements were performed during the follow-up (nbDMARD 481; anti-TNF 4390). This equates to an incidence of SA within 90 days of surgery of $0.7 \%$ in the whole cohort. The adjusted OR for postoperative joint infection in the anti-TNF cohort compared with nbDMARD was 0.8 (0.2-3.5).

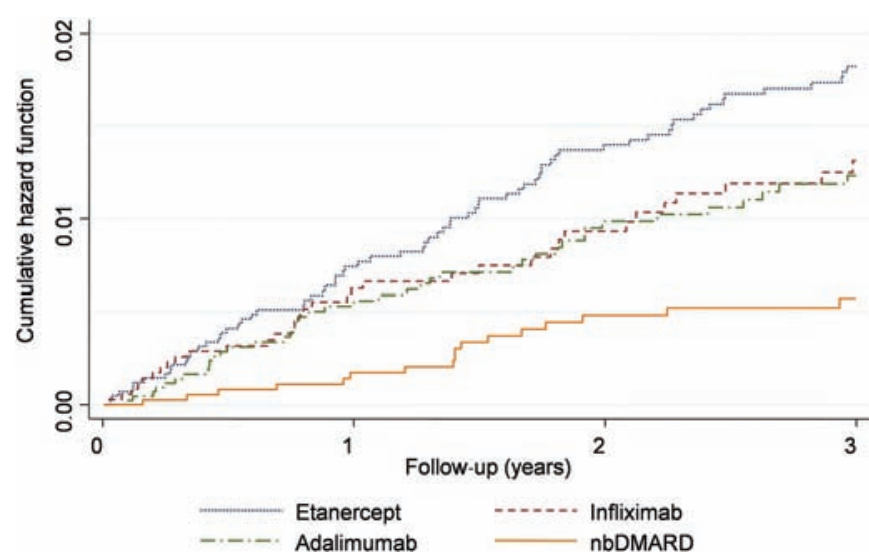

Figure 1 Nelson-Aalen plot comparing nbDMARD and anti-TNF cohorts. Anti-TNF, anti-tumour necrosis factor; nbDMARD, non-biological disease-modifying antirheumatic drug.

\section{DISCUSSION}

Key questions remain unanswered from clinical trial data regarding the safety of anti-TNF therapy, particularly with respect to rare outcomes including SA. This is the first study to specifically examine the rate of SA in patients treated with anti-TNF therapy. We confirmed our primary hypothesis that SA is increased in patients on anti-TNF therapy. However, these results must be interpreted with some important caveats. First, the absolute risk of SA remains very small, with an incident rate of only 4.2 per 1000 pyrs of 


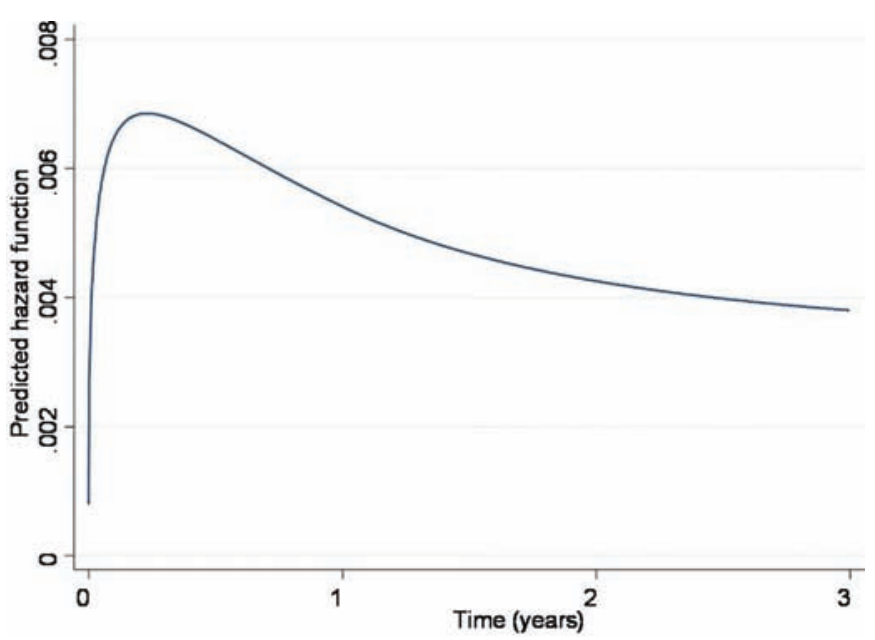

Figure 2 Spline model showing changing risk of septic arthritis over time in the anti-TNF cohort. Anti-TNF, anti-tumour necrosis factor.

Table 3 Baseline patient characteristics

\begin{tabular}{lll}
\hline $\begin{array}{l}\text { Number of patients with } \\
\text { prosthetic joints }\end{array}$ & nbDMARD (n=659) & Anti-TNF (n=2689) \\
\hline Exposure time (years) & 1954 & 12959 \\
$\begin{array}{l}\text { Events } \\
\text { Incidence prosthetic joint SA/1000 }\end{array}$ & 6 & 41 \\
$\begin{array}{l}\text { pyrs (95\% Cl) } \\
\text { Adjusted HR (95\% Cl) }\end{array}$ & Ref & 3.2 (2.3 to 4.3) \\
\hline $\begin{array}{l}\text { *Patients were included in this analysis only if they had a prosthetic joint in situ. } \\
\text { Anti-TNF, anti-tumour necrosis factor; nbDMARD, non-biological disease-modifying } \\
\text { antirheumatic drug; pyrs, patient years. }\end{array}$ &
\end{tabular}

follow-up in the anti-TNF cohort. Second, given the observational nature of this study, it is important to recognise that differences exist between the nbDMARD and anti-TNF cohorts. Any difference in outcome between the two cohorts reflects both the effect of the drug and the inherent differences in patient populations. The biologically treated cohort had, as expected, more severe disease at baseline and this is an important risk factor for the development of SA. We addressed this issue through adjustment using a propensity model. The adjusted analysis continued to show a significant increase in the risk of SA in the anti-TNF cohort (HR 2.3), which reflects the risk attributable to the therapy itself. It is possible that there are unmeasured differences between the cohorts that we are unable to adjust for.

The point estimates showed the highest risk for SA in patients on ETN. However, this was not a hypothesis that we had set out to test. After adjusting for confounders, the 95\% CIs for all three agents overlapped. Year of entry into the study is an important confounder when comparing between agents. We have recently published data from the BSRBR showing that both disease severity and recruitment patterns varied by calendar year. ${ }^{21}$ Therefore, it is not possible to draw firm conclusions about between-drug differences from these data.

As in the general population of RA patients, the most common aetiological organisms were Staphylococcal species. It is important to note that the organisms within the anti-TNF cohort included Listeria and Salmonella, both intracellular pathogens that rarely cause SA. This fits current understanding of the role of TNF in host defence and is in keeping with our previous reports of an increased risk of intracellular organisms (including $M$ tuberculosis) at all sites. ${ }^{9}{ }^{19}$ In the nbDMARD cohort, gram-negative species accounted for $50 \%$ of the organisms, which may reflect the older age of the nbDMARD cohort, whereas in the anti-TNF cohort, only $10 \%$ of the reported species cultured were gram negative. This is of relevance when considering antibiotic choices for patients with RA on anti-TNF therapy. Many guidelines for SA recommend antimicrobial therapy primarily aimed at Staphylococcal species and that lack activity against gram-negative organisms.

The risk of SA appeared to vary over time in the anti-TNF cohort. The finding of a greater risk early on in therapy has been described with other infections in our data as well as data from other cohorts. ${ }^{22}$ There are a number of potential explanations for this early increased risk. First, it may reflect a depletion of susceptible individuals from the exposed cohort. Second, it may reflect a true reduction in risk of joint infection in patients who achieve better control of their RA. However, the data presented here do not allow definite conclusions to be made about these possibilities.

We also addressed the influence of orthopaedic surgery on the risk of SA. The BSR recommends that patients on anti-TNF therapy stop their treatment temporarily for $2-4$ weeks prior to major surgical procedures and do not recommence therapy until wound healing is satisfactory. ${ }^{23}$ Although it is not known with certainty how strictly physicians and patients adhere to these guidelines, in the context of this practice, it is very reassuring to see no evidence of an increased risk of prosthetic joint SA.

In summary, exposure to TNF inhibitor therapy is associated with an increased risk of SA in patients with RA. This risk seen in this study was greatest in the first year of treatment. Joint prosthesis is a key risk factor for infection. Careful vigilance for joint infections in anti-TNF patients remains important, especially in the early months of therapy, with awareness of the potential range of pathogens that may be responsible. Antibiotic guidelines should incorporate this information and consider giving specific advice for patients being actively treated with anti-TNF agents. Current evidence does not support any one anti-TNF agent having a safer profile with regard to SA.

Acknowledgements The authors acknowledge the enthusiastic collaboration of all consultant rheumatologists and their specialist nurses in the UK in providing the data. In addition, the authors acknowledge the support from Dr lan Griffiths (Past) and Professor David Isenberg (Current), Chairs of the BSRBR Management Committee, Professor Gabriel Panayi, Professor David G I Scott, Dr Andrew Bamji and Professor Deborah Bax, presidents of the BSR during the period of data collection, for their active role in enabling the Register to undertake its tasks and to Samantha Peters (CEO of the BSR), Mervyn Hogg, Nia Taylor and members of the BSRBR Scientific Steering Committee. The authors also acknowledge the seminal role of the BSR Clinical Affairs Committee for establishing national biological guidelines and recommendations for such a register. Finally, the authors would like to acknowledge the substantial contribution of Andy Tracey, Katie McGrother and Dr Mark Lunt in database design and manipulation and Professor Alan Silman in his prior role as a principal investigator of the BSRBR.

Funding The BSR commissioned the BSRBR as a UK-wide national project to investigate the safety of biological agents in routine medical practice. BSR receives restricted income from UK pharmaceutical companies, presently Abbott Laboratories, Amgen, Schering Plough (now MSD) and Wyeth Pharmaceuticals (now Pfizer). This income finances a wholly separate contract between the BSR and the University of Manchester, who provide and run the BSRBR data collection, management and analysis services. The principal investigators and their team have full academic freedom and are able to work independently of pharmaceutical industry influence. All decisions concerning analyses, interpretation and publication are made autonomously of any industrial contribution.

Competing interests $\mathrm{MH}$ has received payment for advisory work for Shire, CSL and GSK, as well as receiving financial support for attending conferences from GSK, Octopharma, Biorad, BPL, Phadia, GSK and CSL. All other authors have declared no conflict of interests.

Ethics approval Ethics approval for this study was obtained in December 2000 from the Multicentre Research Ethics Committee (MREC) for the Northwest of England.

Provenance and peer review Not commissioned; externally peer reviewed. 


\section{REFERENCES}

1. Gupta MN, Sturrock RD, Field M. A prospective 2-year study of 75 patients with adult-onset septic arthritis. Rheumatology (Oxford) 2001;40:24-30.

2. Weston VC, Jones AC, Bradbury N, et al. Clinical features and outcome of septic arthritis in a single UK Health District 1982-1991. Ann Rheum Dis 1999;58;4:214-19.

3. Geirsson AJ, Statkevicius S, Vikingsson A. Septic arthritis in Iceland 1990-2002: increasing incidence due to iatrogenic infections. Ann Rheum Dis 2008;67:638-43.

4. Favero M, Schiavon F, Riato L, et al. Rheumatoid arthritis is the major risk factor for septic arthritis in rheumatological settings. Autoimmun Rev 2008;8:59-61.

5. Kaandorp CJ, Van Schaardenburg D, Krijnen P, et al. Risk factors for septic arthritis in patients with joint disease. A prospective study. Arthritis Rheum 1995; 38:1819-25

6. Doran MF, Crowson CS, Pond GR, et al. Frequency of infection in patients with rheumatoid arthritis compared with controls: a population-based study. Arthritis Rheum 2002:46:2287-93.

7. Listing J, Strangfeld A, Kary S, et al. Infections in patients with rheumatoid arthritis treated with biologic agents. Arthritis Rheum 2005;52:3403-12.

8. Askling J, Fored CM, Brandt L, et al. Time-dependent increase in risk of hospitalisation with infection among Swedish RA patients treated with TNF antagonists. Ann Rheum Dis 2007;66:1339-44.

9. Dixon WG, Watson K, Lunt M, et al. Rates of serious infection, including sitespecific and bacterial intracellular infection, in rheumatoid arthritis patients receiving anti-tumor necrosis factor therapy: results from the British Society for Rheumatology Biologics Register. Arthritis Rheum 2006;54:2368-76.

10. Dixon WG, Hyrich KL, Watson KD, et al. Drug-specific risk of tuberculosis in patients with rheumatoid arthritis treated with anti-TNF therapy: results from the BSRBR. Ann Rheum Dis 2010;69:522-28.

11. Strangfeld A, Listing J, Herzer P, et al. Risk of herpes zoster in patients with rheumatoid arthritis treated with anti-TNF-alpha agents. JAMA 2009;301:737-44.

12. Rachapalli S, O'Daunt S. Septic arthritis due to Listeria monocytogenes in a patient receiving etanercept. Arthritis Rheum 2005;52:987.
13. Katsarolis I, Tsiodras S, Panagopoulos P, et al. Septic arthritis due to Salmonella enteritidis associated with infliximab use. Scand J Infect Dis 2005;37:304-5.

14. Elwood RL, Pelszynski MM, Corman LI. Multifocal septic arthritis and osteomyelitis caused by group A Streptococcus in a patient receiving immunomodulating therapy with etanercept. Pediatr Infect Dis J 2003;22:286-8.

15. National Institute for Health and Clinical Excellence. Rheumatoid Arthritis Etanercept and Infliximab. London: NICE, 2001.

16. Hyrich KL, Watson KD, Isenberg DA, et al. The British Society for Rheumatology Biologics Register: 6 years on. Rheumatology (Oxford) 2008;47:1441-3.

17. Kirwan JR, Reeback JS. Stanford Health Assessment Questionnaire modified to assess disability in British patients with rheumatoid arthritis. Br J Rheumatol 1986;25:206-9.

18. Prevoo ML, van't Hof MA, Kuper HH, et al. Modified disease activity scores that include twenty-eight-joint counts. Development and validation in a prospective longitudinal study of patients with rheumatoid arthritis. Arthritis Rheum 1995;38:44-8.

19. Dixon WG, Hyrich KL, Watson KD, et al. Drug-specific risk of tuberculosis in patients with rheumatoid arthritis treated with anti-TNF therapy: results from the British Society for Rheumatology Biologics Register (BSRBR). Ann Rheum Dis 2010;69:522-8.

20. Curtis LH, Hammill BG, Eisenstein EL, et al. Using inverse probability-weighted estimators in comparative effectiveness analyses with observational databases. Med Care 2007:45(10 Suppl 2):S103-7.

21. Hyrich KL, Watson KD, Lunt $\mathrm{M}$, et al. Changes in disease characteristics and response rates among patients in the United Kingdom starting anti-tumour necrosis factor therapy for rheumatoid arthritis between 2001 and 2008. Rheumatology (Oxford) 2011;50:117-23.

22. Askling J, Dixon W. The safety of anti-tumour necrosis factor therapy in rheumatoid arthritis. Curr Opin Rheumatol 2008;20:138-44.

23. Ledingham J, Deighton C. Update on the British Society for Rheumatology guidelines for prescribing TNFalpha blockers in adults with rheumatoid arthritis (update of previous guidelines of April 2001). Rheumatology (Oxford) 2005; 44:157-63. 